\begin{tabular}{lc} 
Sharif University of Technology & Scientia Iranica \\
SCIENTIA & Transactions F: Nanotechnology \\
I RAN ICA & http://scientiairanica.sharif.edu \\
\hline
\end{tabular}

\title{
Plasmonic circular dichroism study of gold nanorod-quadruplex nanobioconjugates
}

\author{
Z. Bagheri ${ }^{\mathrm{a}}$, B. Ranjbar ${ }^{\mathrm{b}, \mathrm{c}, *}$, A. Azizi ${ }^{\mathrm{d}}$, H. Latifie ${ }^{\mathrm{e}}$ M. Ismail Zibaii ${ }^{\mathrm{e}}$ and \\ T. Tohidi Moghadam ${ }^{\mathrm{c}}$ \\ a. Protein Research Center, Shahid Beheshti University G.C., Velenjak, Tehran, Iran. \\ b. Department of Biophysics, Faculty of Biological Sciences, Tarbiat Modares University, Tehran, P.O. Box 154-14115, Iran. \\ c. Department of Nanobiotechnology, Faculty of Biological Sciences, Tarbiat Modares University, Tehran, P.O. Box 154-14115, Iran. \\ d. Department of Bioscience and Biotechnology, Malek-Ashtar University of Technology, Tehran, Iran. \\ e. Laser and Plasma Research Institute, Shahid Beheshti University G.C., Velenjak, Tehran, Iran.
}

Received 1 August 2014; received in revised form 5 September 2016; accepted 28 August 2017

\author{
KEYWORDS \\ Parallel and \\ antiparallel G- \\ quadruplexes; \\ Surface plasmon \\ resonance; \\ Plasmonic circular \\ dichroism; \\ Gold nanorod.
}

\begin{abstract}
Circular dichroism spectroscopy is a simple way to study G-quadruplex structure and is very useful for monitoring the conformational changes in G-quadruplex structure induced by modifications to the environment. Plasmonic nanoparticles with Localized Surface Plasmon Resonance (LSPR) can create strong electromagnetic fields at the surface of plasmonic metals, which remarkably influence the optical properties of molecules. Plasmonic CD is a new CD signal, which originates from the dipole-dipole interactions between surface plasmons of NPs, and chiral biomolecules have received interest in various research fields of nanotechnology. In this paper, we study the interactions between Gold NanoRods (GNR) and two types of G-quadruplex (parallel and antiparallel) to monitor the alterations in DNA conformation and plasmonic CD signal upon formation of GNR-quadruplex nanobioconjugate. The results from this study indicate the plasmonic CD signals in visible regions are more sensitive than Far-UV CD signals to detect the spatial conformational state of G-quadruplex-GNR nanobioconjugate.
\end{abstract}

(C) 2018 Sharif University of Technology. All rights reserved.

\section{Introduction}

Plasmonic Nanoparticles (NPs) have offered a variety of applications in biosensing technologies. Among metal nanoparticles, gold nanostructures with rod morphology have drawn great interest due to their shapedependent optoelectronic properties [1-3]. Up to now, there have been many reports about different applications of DNA-gold nanorods (GNR) bioconjugation in biological and biochemical sensing [4]. Although the mechanism of interaction between GNRs and

*. Corresponding author. Tel.: +982182884482 E-mail address: ranjbarb@modares.ac.ir (B. Ranjbar)

doi: $10.24200 /$ sci. 2017.4431 biomolecules has not yet been fully understood, the rod morphology of GNRs might induce some changes in the biomolecules' structure [5]. In order to investigate the secondary structure alterations of biomolecules after bioconjugation with GNRs, Circular Dichroism (CD) is a proper technique. In biomolecules, CD signals typically appear in the UV range while the intensity of CD signals in the visible region is weak. The intensity of $\mathrm{CD}$ response in the visible regions could be enhanced by interactions between the chiral biomolecules and plasmonic NPs due to interactions between surface plasmons of NPs and chiral biomolecules [6].

Nucleic acid aptamers are generally obtained by in vitro selection. Some of them have G-rich consensus sequences with ability to be folded in the four-stranded structures known as G-quadruplexes. The repetitive 


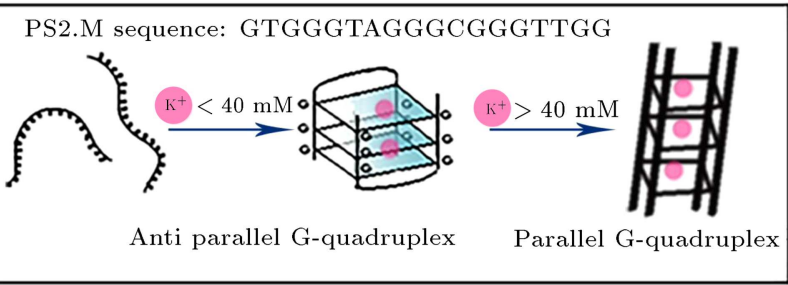

Scheme 1. Different geometries of PS2.M G-quadruplex in the presence of $\mathrm{K}^{+}$and $\mathrm{Pb}^{2+}$ cations.

part in this structure is a square plane called G-quartet, consisting of 4 guanines held together by guanine Hoogsteen hydrogen bonding [7]. In fact, G-quartet is a broad aromatic surface, which is highly prone to $\pi-\pi$ stacking interaction between adjacent surfaces [7]. Apart from the order and number of guanines in the G-quadruplex prone oligonucleotide, quadruplex inducing factors, i.e. metal ions, alcohol, etc., are also parameters that cause variations in quadruplex families [8].

It is commonly accepted that the conformation of a G-quadruplex structure (parallel, antiparallel, or mixed) can be roughly assigned from the position and magnitude of the CD bands. Hence, a positive band at $260 \mathrm{~nm}$ is commonly assigned to a parallel conformation, whereas a positive band at $295 \mathrm{~nm}$ and a negative band at $260 \mathrm{~nm}$ are indicative of an antiparallel conformation [8-9]. PS2.M is 18 nucleotides guanine-rich sequence capable to form anti-parallel and parallel G-quadruplexes in the presence of various concentrations of metal ions (Scheme 1) [10].

Herein, an alteration in secondary structure and plasmonic CD signals of two types of G-quadruplex (parallel and antiparallel) upon interactions with GNRs has been monitored via circular dichroism spectroscopy.

\section{Material and methods}

\subsection{Material and reagents}

PS2.M oligonucleotide with sequence as:

\section{GTGGGTAGGGCGGGTTGG}

was procured from Bioneer chemical Co. Lead (II) acetate trihydrate $(\mathrm{PbAc})$, potassium chloride $(\mathrm{KCl})$, 4-(2-hydroxyethyl) piperazine-1-ethanesulfonic acid hemisodium salt (HEPES), $\mathrm{HAuCl}_{4} 3 \mathrm{H}_{2} \mathrm{O}$ $(99.9 \%), \mathrm{NaBH}_{4}(99 \%)$, ascorbic acid (99\%), hexadecyltrimethylammonium bromide (CTAB) (99\%), and $\mathrm{AgNO}_{3}$ (99\%) were purchased from Sigma.

\subsection{G-Quadruplex formation protocol}

Formation of G-quadruplex occurred in special buffer $25 \mathrm{mM}$ HEPES, $100 \mathrm{mM} \mathrm{NaCl}$, and various concentrations of $\mathrm{KCl}$ or $\mathrm{PbAc}$ as the stabilizer agent for four stranded structures. To initiate formation of Gquadruplex structure, PS2.M was heated at $80^{\circ} \mathrm{C}$ for $10 \mathrm{~min}$ in various concentrations of $\mathrm{K}^{+}$or $\mathrm{Pb}^{2+}$ solutions and gradually cooled down to room temperature.

\subsection{Preparation of Au seeds and nanorods}

Short gold nanorods were synthesized via sequential seed mediated growth method. The protocol has been formerly described by a number of authors [11,12]. The procedure started with preparation of small spherical gold nanoparticles of sizes below $5 \mathrm{~nm}$ (to be used as seeds). In a test tube, $250 \mu \mathrm{L}$ of an aqueous 0.01 $\mathrm{M}$ solution of $\mathrm{HAuCl}_{4} \cdot 3 \mathrm{H}_{2} \mathrm{O}$ was added to $7.5 \mathrm{~mL}$ of a $0.095 \mathrm{M}$ CTAB solution. Immediately, $600 \mu \mathrm{L}$ of aqueous $0.01 \mathrm{M}$ ice-cold $\mathrm{NaBH}_{4}$ solution was added and the reactants were mixed by rapid inversion for $2 \mathrm{~min}$. Meanwhile, evolved gas was allowed to escape at the time of mixing. The solution developed a pale brownyellow color. The test tube was then kept at room temperature. It took the minimum of $2 \mathrm{~h}$ for seed particles to grow in the solution. Appropriate quantities of CTAB solution, $\mathrm{HAuCl}_{4}, \mathrm{AgNO}_{3}$, ascorbic acid, and seed solutions were prepared for the growth stage. In a typical experiment, $9.5 \mathrm{~mL}$ of $0.095 \mathrm{M}$ CTAB, $400 \mathrm{~L}$ of $0.01 \mathrm{M} \mathrm{HAuCl}_{4} .3 \mathrm{H}_{2} \mathrm{O}$, and $60 \mu \mathrm{L}$ of $0.01 \mathrm{M} \mathrm{AgNO}_{3}$ solutions were added respectively and mixed very gently. At this stage, the solution gave an intense color. The procedure was continued by addition of $64 \mu \mathrm{L} 0.10 \mathrm{M}$ ascorbic acid. Ascorbic acid with its key role in further reduction in gold changed the appearance to a colorless solution. Finally, $40 \mu \mathrm{L}$ of seed particles were added. The reaction mixture in the test tube was shaken gently for $10 \mathrm{~s}$ and kept undisturbed for $3 \mathrm{~h}$. Intense purple-brown color started to appear within a couple of minutes.

\subsection{Purification of gold nanorods}

Gold nanorods were prepared in the presence of excess amount of CTAB as a cationic surfactant. In order to remove excess of CTAB and to separate unreacted gold ions from the solution, samples were purified with two rounds of centrifugation (12,000 rpm, $6 \mathrm{~min})$. The first-round supernatant was decanted and gold nanorod sample was diluted with deionized water; in the second round, dilution was made with phosphate buffer to keep $\mathrm{pH}$ adjusted at 6.2 .

\subsection{TEM and $U V$-Vis characterization}

Transmission Electron Microscopy (TEM) study was performed with a TE 2000 Ziess electron microscope. Prior to TEM preparation, the sample was purified by two rounds of centrifugation and re-dispersion in DI water. Dilute sample was deposited on a carbon coated copper grid and left undisturbed for solvent evaporation. Gold nanorods were also characterized by UV-Vis absorption spectrophotometer (Cary 100). The 
spectra were recorded within 400-900 $\mathrm{nm}$ wavelength range.

\subsection{Circular dichroism spectroscopy}

The interaction of GNR with quadruplex was monitored in two different regions with circular dichroism spectroscopy. The spectra were recorded from 200 to $320 \mathrm{~nm}$ (Far-UV) for studying the influence of GNR on the conformational changes of G-quadruplexes. To investigate the conjugation of GNR with quadruplex, plasmonic circular dichroism was recorded in visible region (400-900 nm). Circular dichroism and plasmonic CD experiments were carried out via J-715 spectropolarimeter; CD data were smoothed with J-715 software. The CD spectra were obtained in a cell with $0.1 \mathrm{~cm}$ path length at room temperature. The background CD spectrum of buffer was subtracted for each sample.

\section{Results and discussions}

\subsection{Conformational characterization of PS2.M G-quadruplexes}

Circular dichroism spectroscopy is a sensitive way to study G-quadruplex structure and is very useful for monitoring the conformational changes in Gquadruplex structure induced by modifications of the environment [8]. Since CD spectroscopy is very sensitive to base stacking [7-9], different configurations of quadruplex (such as parallel, antiparallel, and hybrid forms) have their specific characteristic of $\mathrm{CD}$ spectrum [8,9]. Figure 1 depicts the CD spectra of PS2.M in various concentrations of potassium ion. In the Far-UV CD spectra of PS2.M in the presence of low potassium ion concentrations, $295 \mathrm{~nm}$ positive and $260 \mathrm{~nm}$ negative bands are well representative of antiparallel quadruplex, and $260 \mathrm{~nm}$ positive and $240 \mathrm{~nm}$ negative bands of PS2.M in the presence of

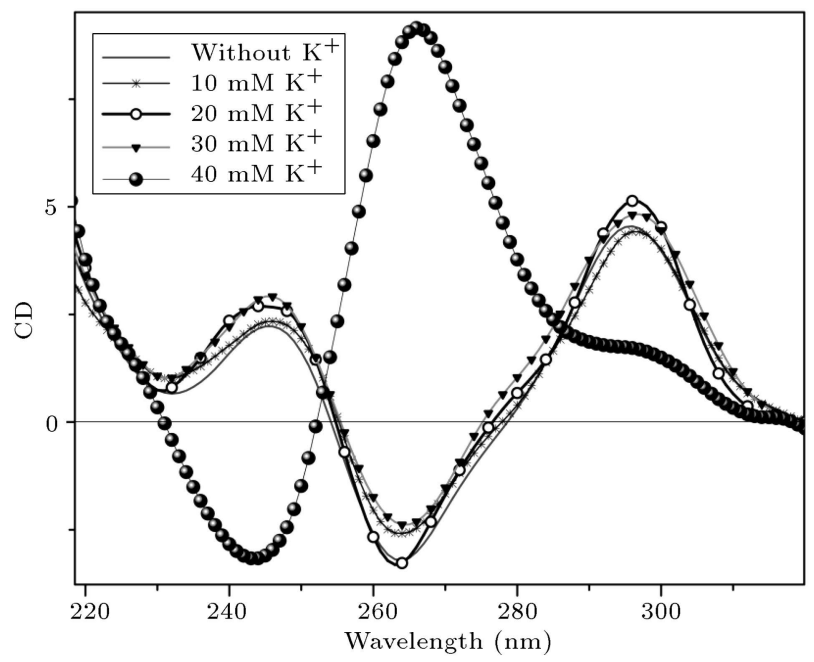

Figure 1. CD spectra of PS2.M in various concentrations of $\mathrm{K}^{+}$.
$40 \mathrm{mM} \mathrm{K}^{+}$are characteristics of parallel conformation.

\subsection{Characterization of gold nanorods}

Gold nanorod samples were characterized by transmission electron microscopy and UV-Vis spectroscopy. Depending on the polarization of the incident, light electron oscillation can occur in two directions [13]. The excitation of surface plasmon oscillation along the short axis induces an absorption band in the visible region $(520 \mathrm{~nm})$. When the surface plasmon of the nanostructure oscillates along the other axis, an absorption band of much stronger intensity appears at a longer wavelength $(712 \mathrm{~nm})$. These absorption peaks could be referred to as the transverse and longitudinal plasmon resonance bands, respectively, being characteristic of the rod morphology of gold nanostructures (Figure 2). Transmission electron microscopy also confirms the formation of gold nanorods (Figure 3 ).

\subsection{Circular dichroism spectroscopy of the interaction between GNR and quadruplex}

Figure 4 indicates far-UV CD spectra of G-quadruplex

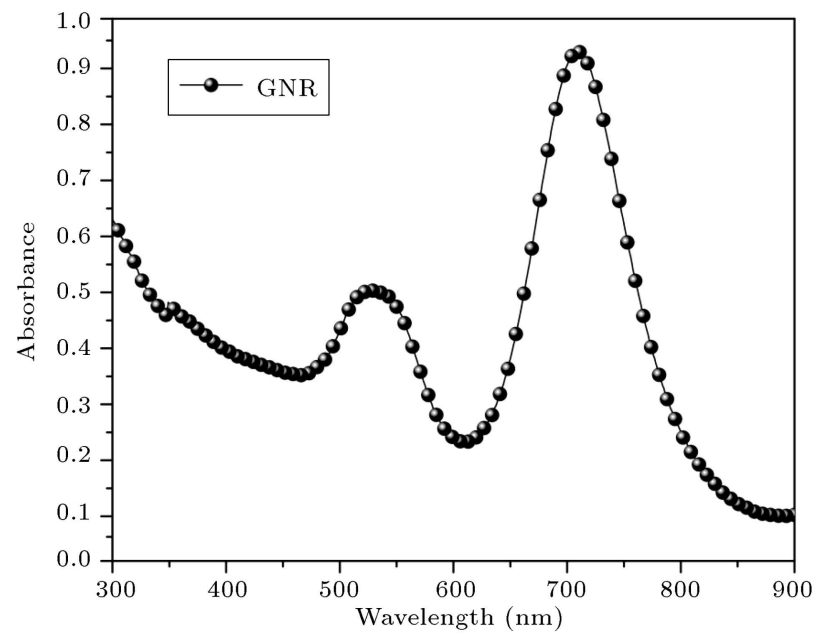

Figure 2. Surface plasmon resonance of gold nanorods.

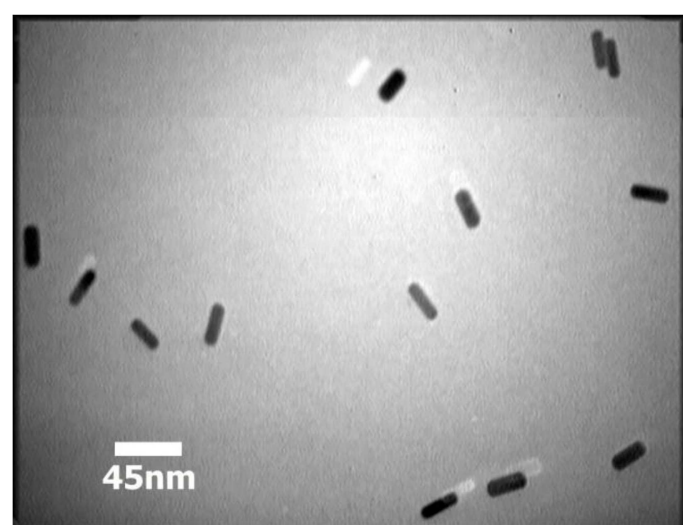

Figure 3. Transmission electron micrograph of purified gold nanorods. 


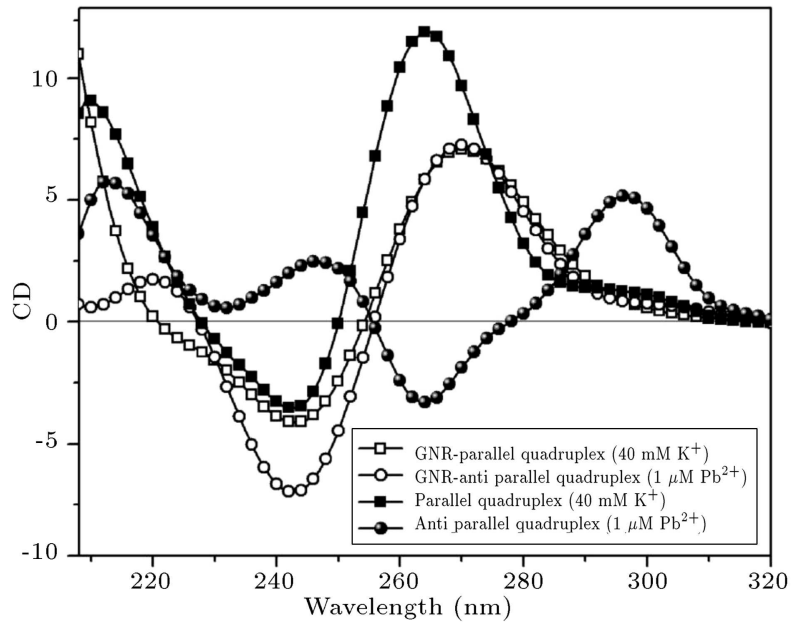

Figure 4. Circular dichroism spectra of parallel quadruplex (with $40 \mathrm{mM} \mathrm{K}^{+}$) and antiparallel quadruplex (with $1 \mathrm{uM} \mathrm{Pb}^{2+}$ ) before and after interaction with GNRs.

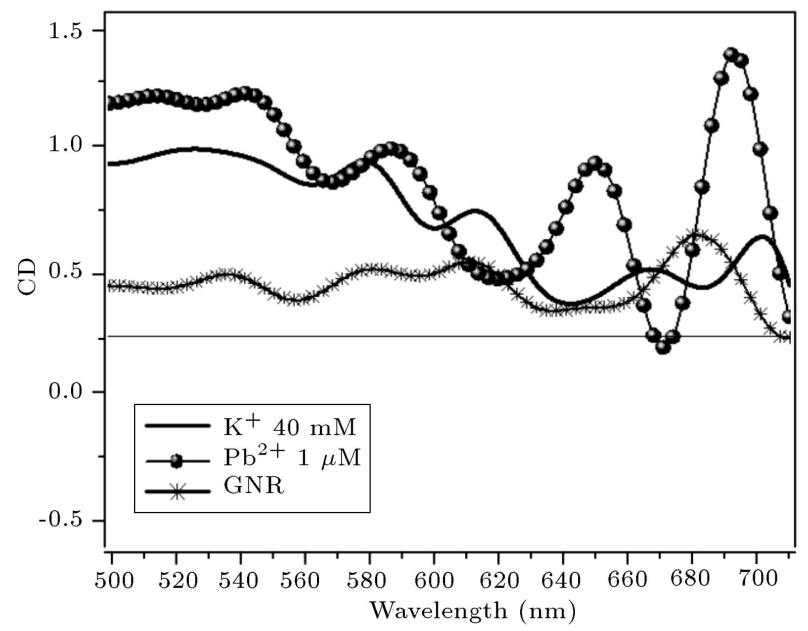

Figure 5. Plasmonic circular dichroism spectra of interaction between GNRs and parallel quadruplex (with $40 \mathrm{mM} \mathrm{K}^{+}$) or antiparallel quadruplex (with $1 \mathrm{uM} \mathrm{Pb}^{2+}$ )

before and after interaction with GNRs, in the presence of $40 \mathrm{mM} \mathrm{K}^{+}$and $1 \mu \mathrm{M} \mathrm{Pb}^{2+}$.

Before its interactions with GNRs in the presence of $\mathrm{KCl}$, the G-quadruplex shows extremely significant parallel CD signal at $260 \mathrm{~nm}$, while in the presence of $\mathrm{PbAc}$, there are two $\mathrm{CD}$ signals with apposite sings, positive band at $295 \mathrm{~nm}$ and negative band at $260 \mathrm{~nm}$, which are responsible for the antiparallel conformational state of quadruplex. After interaction with GNRs, both parallel and antiparallel conformations are modified and the new CD signals, similar to single stranded DNA, appear. Figure 5 shows plasmonic circular dichroism spectra of GNRs, before and after the interactions with G-quadruplex in the presence of $40 \mathrm{mM} \mathrm{K}^{+}$or $1 \mu \mathrm{M} \mathrm{Pb}^{2+}$.

GNRs show two weak plasmonic CD signals that appear at $540 \mathrm{~nm}$ and $680 \mathrm{~nm}$, respectively. These signals could be responsible for transverse and lon- gitudinal SPR bands of GNRs, respectively. After interactions between GNRs and G-quadruplex, in the presence of $\mathrm{K}^{+}$(parallel quadruplex), the intensity of transverse plasmonic CD band increases and the longitudinal plasmonic CD peak shifts to longer wavelengths. In the presence of $\mathrm{Pb}^{2+}$, not only the intensity of both transversal and longitudinal plasmonic CD peaks increases, but also the shape and position of the peak are modified.

\section{Conclusion}

The present study reports the circular dichroism of interactions between GNRs and PS2.M quadruplexes within two different wavelength regions. Comparison of CD signals in the Far-UV region (200-320 nm) indicated that the conformation of quadruplex changed after interaction with GNRs. The changes were noticed to be approximately similar for both parallel and antiparallel quadruplex structures. Upon interaction of GNRs with quadruplex structures, intensity of CD signal increased in the visible region, which could be attributed to the coupling of surface plasmon resonance of GNRs and the chiral signal of DNA. Furthermore, after the interaction, parallel and antiparallel quadruplex structures developed different CD spectra in this region. Circular dichroism study of G-quadruplex-GNR nanobioconjugate system revealed that plasmonic CD signals in the visible regions were more sensitive to detect the spatial conformational state of G-quadruplex than Far-UV CD signals.

\section{Acknowledgment}

The authors would like to thank the research council of Tarbiat Modares University for financial support of this work.

\section{References}

1. Zhu, Xinli, Yang Zhang, Jiasen Zhang, Jun Xu, Yue Ma, Zhiyuan Li, and Dapeng Yu. "Ultrafine and smooth full metal nanostructures for plasmonics", Advanced Materials, 22(39), pp. 4345-4349 (2010).

2. Kuzyk, Anton, Robert Schreiber, Zhiyuan Fan, Günther Pardatscher, Eva-Maria Roller, Alexander Higele, Friedrich C. Simmel, Alexander O. Govorov, and Tim Liedl. "DNA-based self-assembly of chiral plasmonic nanostructures with tailored optical response", Nature, 483(7389), pp. 311-314 (2012).

3. Haes, Amanda, J., Christy, L. Haynes, Adam, D., McFarland, George, C. Schatz, Richard, P. Van Duyne, and Shengli Zou. "Plasmonic materials for surfaceenhanced sensing and spectroscopy", MRS Bulletin, 30(5), pp. 368-375 (2005).

4. Tan, Shawn J., Michael J. Campolongo, Dan Luo, and Wenlong Cheng. "Building plasmonic nanostructures 
with DNA", Nature Nanotechnology, 6(5), pp. 268-276 (2011).

5. Park, Sung Yong, Julianne M. Gibbs-Davis, SonBinh T. Nguyen, and George C. Schatz. "Sharp melting in DNA-linked nanostructure systems: thermodynamic models of DNA-linked polymers", The Journal of Physical Chemistry B, 111(30), pp. 8785-8791 (2007).

6. Azizi, A., Ranjbar, B., Moghadam, T., and Bagheri, Z. "Plasmonic circular dichroism study of DNA-Gold nanoparticles bioconjugates", Plasmonics, 9(2), pp. 273-281 (2014).

7. König, Sebastian, L.B., Amanda, C. Evans, and Huppert, J.L. "Seven essential questions on Gquadruplexes", Biomolecular Concepts, 1(2), pp. 197213 (2010).

8. Kypr, Jaroslav, Iva Kejnovská, Renčiuk, D., and VorlÍčková, M. "Circular dichroism and conformational polymorphism of DNA", Nucleic Acids Research, 37(6), pp. 1713-1725 (2009).

9. Jaumot, Joaquim, and Raimundo Gargallo "Experimental methods for studying the interactions between G-quadruplex structures and ligands", Current Pharmaceutical Design, 18(14), pp. 1900-1916 (2012).

10. Liu, Wei, Hong Zhu, Bin Zheng, Sheng Cheng, Yan Fu, Wei Li, Tai-Chu Lau, and Haojun Liang. "Kinetics and mechanism of G-quadruplex formation and conformational switch in a G-quadruplex of PS2. M induced by Pb2+", Nucleic Acids Research, 40(9), pp. 4229-4236 (2012).

11. Moghadam Tohidi, T., Ranjbar, B., Khajeh, K.H., Etezad, S.M., Khalifeh, K.H., and Ganjalikhany, M.R. "Interaction of lysozyme with gold nanorods: conformation and activity investigations", International Journal of Biological Macromolecules, 49(4), pp. 629636 (2011).

12. Sau, Tapan K., and Catherine J. Murphy. "Seeded high yield synthesis of short $\mathrm{Au}$ nanorods in aqueous solution", Langmuir, 20(15), pp. 6414-6420 (2004).

13. Huang, Xiaohua, Svetlana Neretina, and Mostafa A. El-Sayed "Gold nanorods: from synthesis and properties to biological and biomedical applications", Advanced Materials, 21(48), pp. 4880-4910 (2009).

\section{Biographies}

Zeinab Bagheri received the BSc degree in Cellular and Molecular Biology from Tehran University in 2007. She obtained her MSc and PhD degrees from Tarbiat Modares University, Tehran, Iran, in 2009 and 2014, both in Biophysics. She is currently an Assistant professor in Protein Research Center at Shahid Beheshti University. Her research interests include fiber optic biosensors, functional DNA nanostructures, and circular dichroism spectroscopy.

Bijan Ranjbar received the BSc degree in Chemistry from University of Isfahan and MSc in Biochemistry from Institute of Biochemistry and Biophisycs (IBB) at University of Tehran. He received the $\mathrm{PhD}$ degree in Biophysics from Moscow Institute of Physics and Technology, and Engelhardt Institute of Molecular Biology. He joined Tarbiat Modares University in October 1997, where he is currently a Full Professor of Biophysics in the Department of Biophysics. His current research interests focus on biophysical studies of protein, DNA, dnzyme, and hybrid DNA nanostructures with promising applications in designing nanobiosensors and drug nanocarrier systems.

Azadeh Azizi was born in Khoramabad, Iran, in 1986. She received BSc degree in Biology from Lorestan University, Khoramabad, Iran, in 2008 and MSc and PhD degrees in Biophysics from the Institute of Biological Science at Tarbiat Modares University, Tehran, Iran, in 2010 and 2014, respectively. In 2015, she joined the Department of Bioscience and Biotechnology at MalekAshtar University of Technology, Tehran, Iran.

Hamid Latifi received the BSc degree from California State University in Harvard, and the MSc and PhD degrees from New-Mexico State University, Las Cruces, in 1989, all in Physics, working on interaction of high-energy laser beam aerosols. He was a postdoctoral researcher at Colorado State University in Fort Collins, working on development of sodium Lidar for mesospheric temperature measurement. He joined the Physics Faculty of Shahid Beheshti University (formerly, National University), Tehran, Iran, in 1991, where he is currently a Professor of Physics at Laser and Plasma Research Institute. His current interest includes diode pumped solid state laser, RF-excited CO2 lasers, optical nondestructive testing, and fiberoptic sensors.

Mohammad Ismail Zibaii received the MSc and $\mathrm{PhD}$ degrees in Photonics from Laser and Plasma Research Institute, Shahid Beheshti University, Tehran, Iran, in 2011. His PhD degree focused on research in fiber-optic biosensors. He is currently an Assistant Professor of Photonics at Laser and Plasma Research Institute of Shahid Beheshti University. His research interest includes neurophotonics, optogenetics and development of fiber-optic sensors and label-free fiber optic biosensors for DNA-Drug interaction, bacteria, and protein in low concentration.

Tahere Tohidi Moghadam is an Assistant Professor of Nanobiotechnology at Tarbiat Modares University. Her research interests are mainly focused on plasmonic gold nanorods with theranostic applications, and she is currently completing a research project on assembling these nanostructures on amyloid fibril scaffolds to attain conductive nanowires. Her recent publications 
on diagnostic applications of gold nanoparticles with surface plasmon resonance property (SPR) include "Heat Induced Aggregation of Gold Nanorods for Visual Detection of Lysozyme" in Talanta, 2015, and
"Development of a Label-free SPR Sensor for Detection of Matrixmetalloproteinase- 9 by Antibody Immobilization on Carboxymethyldextran Chip" in Biosensors and Bioelectronics, 2016. 